\title{
¿Cuántas competencias transversales podemos diferenciar en un auto-diagnóstico?
}

Marin-Garcia, Juan A. ${ }^{a}$, Atarés, Lorena ${ }^{\mathrm{b}}$; Aznar-Mas, Lourdes E. ${ }^{c}$

a ROGLE. Dpto. de Organización de Empresas. Universitat Politècnica de València. Camino de Vera S/N 46021 Valencia. ${ }^{a}$ jamarin@omp.upv.es, ${ }^{\mathrm{b}}$ julien.maheut@gmail.com and ${ }^{\mathrm{c}}$ jugarsa@omp.upv.es. ${ }^{\mathrm{b}}$ Departamento de Tecnología de Alimentos. Universidad Politécnica de Valencia. Camino de Vera S/N 46022 Valencia. loathue@tal.upv.es

c EICE IEMA, Dpto. Lingüística Aplicada, UPV, laznar@idm.upv.es,

\section{Abstract}

The UPV has identified 12 soft-skills based on the lists of EURACE, ANECA and ABET. However, are they sufficiently independent or overlapping? Our work explores by factor analysis how many dimensions can be obtained sufficiently different from each other. To do this we will analyze a sample of students and professionals with data based on a self-assessment

Keywords: :self-assessment; soft skills; higher education; practitioners

\section{Resumen}

La UPV ha identificado 12 competencias transversales basadas en los listados de EURACE, ANECA y ABET. Sin embargo, ¿son suficientemente independientes o están solapadas?

Nuestro trabajo explora mediante análisis factorial cuántas dimensiones se pueden obtener suficientemente diferentes entre sí. Para ello analizaremos una muestra de alumnos y de profesionales con datos basados en un autodiagnóstico.

Palabras clave: calificación; desempeño de estudiantes; educación universitaria; evaluación sumativa; competencias transversales; comparación pareada

\section{Introducción}

Las competencias transversales (soft skills) han sido incorporadas a las memorias y planes de estudios de los grados universitarios de ingeniería, u otros grados, a instancias de los requisitos de diferentes normativas españolas (RD 1393/2007, 861/2010, 1027/2011; Orden CIN/311/2009, CIN/351/2009; resolución 12977/2009) y de las agencias nacionales o internacionales de acreditación: ANECA/AVAP (Argos \& Ezquerra, 2014; Miró \& Jaume i Capó, 2010; Tovar \& Castro, 2007), EURACE (Patil \& Codner, 2007; Tovar \& Castro, 2007) y ABET (McGourty et al., 2002; Passow, 2012; Shuman et al., 2005; Terry et al., 2002; Weiss \& Aldridge, 2002; Williams, 2002).

El grado de solape o congruencia entre las competencias elegidas por las diferentes agencias es bastante bajo. En unos casos, se requieren unas competencias que otros sistemas no contemplan. En otros, competencias con nombres similares tienen definiciones radicalmente diferentes. Por último, también se da el caso de que definiciones similares tienen nombres o rótulos diferentes en cada uno de los sistemas.

En cualquier caso, la Universitat Politècnica de València (UPV) realizó un proceso de integración de las propuestas de las tres agencias (ANECA/AVAP, ABET y EURACE) que culminó con un listado de 12 competencias transversales (más una 13 $3^{\mathrm{a}}$ que no es transversal a todos los grados sino que son competencias específicas del grado), que pretenden dar cobertura completa a los requisitos de las tres agencias (http://www.upv.es/contenidos/COMPTRAN/info/955709normalc.html):

- CT-01. Comprensión e integración

- CT-02. Aplicación y pensamiento práctico

- CT-03. Análisis y resolución de problemas 
- CT-04. Innovación, creatividad y emprendimiento

- CT-05. Diseño y proyecto

- CT-06. Trabajo en equipo y liderazgo

- CT-07. Responsabilidad ética, medioambiental y profesional

- CT-08. Comunicación efectiva

- CT-09. Pensamiento crítico

- CT-10. Conocimiento de problemas contemporáneos

- CT-11. Aprendizaje permanente

- CT-12. Planificación y gestión del tiempo

Sin embargo, hasta la fecha, no hemos encontrado ningún estudio empírico que compruebe si estas 12 competencias son elementos independientes y diferenciados, o si todas ellas no son más que reflejo de un constructo subyacente o meta-competencia de ingeniería que las resume todas. Tampoco hemos encontrado trabajos que comparen la estructura dimensional de estas competencias en estudiantes universitarios y en profesionales de empresa.

\section{Objetivos}

Obj. 1) Comprobar la correlación que existe entre 9 de estas competencias transversales

Obj. 2) Analizar la estructura subyacente de este conjunto de competencias e identificar los constructos latentes en los que se podrían agrupar estas competencias

Obj. 3) Comprobar si la estructura es similar en una muestra de estudiantes y otra de profesionales. Hemos recogido datos de una muestra de profesionales porque las competencias transversales se han incluido en los procesos de verificación de títulos porque son aspectos demandados por las empresas y están asociados al éxito de los profesionales. De modo que queremos analizar si las relaciones entre las competencias que aparecen en los estudiantes son parecidas a las que se obtienen con profesionales

\section{Desarrollo de la innovación}

Los datos se tomaron en mayo de 2016 mediante un cuestionario web.

Los datos de estudiantes proceden de alumnos de la titulación de Grado en Ingeniería de Organización Industrial. De los 312 alumnos matriculados en la titulación respondieron 128 (41\% de tasa de respuesta).

Los datos de profesionales provienen las plantas de valencia de dos empresas multinacionales del sector de la electrónica donde la innovación es su característica distintiva. En cada planta los directores de recursos humanos seleccionaron a 30 y 29 personas incorporadas al programa de gestión de talento innovador. De los 59 trabajadores seleccionados, 47 respondieron ( $80 \%$ de tasa de respuesta).

Las definiciones de las competencias para construir los mono-ítems se han obtenido a partir de las definiciones publicadas en la página web http://www.upv.es/contenidos/COMPTRAN/info/957657normalc.html (las CT-04 y CT-06 se han desdoblado en dos para responder a los dos matices diferenciadores de la competencia base). Se seleccionaron 9 de las 12 competencias transversales, dejando de lado las que los autores consideraron que era complicado construir una definición de una frase que representara de manera unívoca y completa la competencia (ver tabla 1).

Tabla 1 . Definición de las variables

\begin{tabular}{|l|l|l|}
\hline CT & Código & Descripción \\
\hline CT-01 & A_compre & [Comprender e integrar el conocimiento del que dispones] \\
\hline CT-02 & A_aplic & $\begin{array}{l}\text { [Aplicar los conocimientos a la práctica, atendiendo a la } \\
\text { información disponible, y estableciendo el proceso a seguir } \\
\text { para alcanzar los objetivos con eficacia y eficiencia.] }\end{array}$ \\
\hline
\end{tabular}

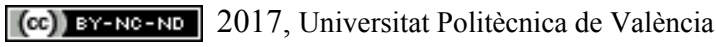




\begin{tabular}{|l|l|l|}
\hline CT-03 & A_proble & $\begin{array}{l}\text { [Analizar y resolver problemas de forma efectiva, } \\
\text { identificando y definiendo los elementos significativos que } \\
\text { los constituyen.] }\end{array}$ \\
\hline CT-04 & A_empre & $\begin{array}{l}\text { [Innovar para responder satisfactoriamente y de forma } \\
\text { original, a las necesidades y demandas personales, } \\
\text { organizativas y sociales con una actitud emprendedora. }\end{array}$ \\
\hline CT-04 & A_creat & $\begin{array}{l}\text { [pensar más allá de las ideas tradicionales, normas, patrones } \\
\text { orelaciones yenerar o adaptar alternativas, ideas, productos, } \\
\text { procesos, métodos o servicios, independientemente de su } \\
\text { viabilidad o valor añadido }]\end{array}$ \\
\hline CT-05 & A_project & $\begin{array}{l}\text { [Diseñar y evaluar una idea de manera eficaz hasta } \\
\text { concretarla en un proyecto] }\end{array}$ \\
\hline CT-06 & A_leader & $\begin{array}{l}\text { [Liderar equipos de forma efectiva para la consecución de } \\
\text { objetivos comunes] }\end{array}$ \\
\hline CT-06 & A_teamw & $\begin{array}{l}\text { [trabajar eficazmente con otros en grupo] } \\
\text { [Comunicarse de manera efectiva, tanto de forma oral como } \\
\text { escrita] }\end{array}$ \\
\hline CT-08 & A_commu & $\begin{array}{l}\text { [analizar asuntos con un propósito, evaluar las ventajas o } \\
\text { desventajas, y estimar los riesgos asociados] }\end{array}$ \\
\hline CT-09 & A_criti & $\begin{array}{l}\text { [Planificar adecuadamente el tiempo disponible y programar } \\
\text { las actividades necesarias para alcanzar los objetivos, tanto } \\
\text { académico-profesionales como personales. }\end{array}$ \\
\hline CT-12 & A_time \\
\hline
\end{tabular}

Tanto en la muestra de alumnos, como en la de profesionales, las variables se mostraban a cada persona en orden aleatorio y la pregunta introductoria fue: "Toma como referencia lo que has demostrado en un contexto donde necesitas ser innovador o ayudar a un equipo a serlo (bien en un puesto de trabajo en tu organización o en tus tareas como estudiante). Cómo consideras que es tu capacidad para ..."

En las dos sub-muestras (alumnos y profesionales) las escalas de respuesta usaban similares anclas verbales. Sin embargo la escala de alumnos era una escala discreta con 5 niveles de respuesta (1 a 5), más un "no demostrado" (valor 0) (Figura 1). Para los profesionales las escalas iban desde Necesita mejorarla mucho (valor 1) hasta Excelente (valor 100), con la posibilidad de marcar “(..)" si no podían valorar esa capacidad (valor 0) (Figura 2).

Figura 1. Ejemplo de parte del cuestionario para alumnos universitarios

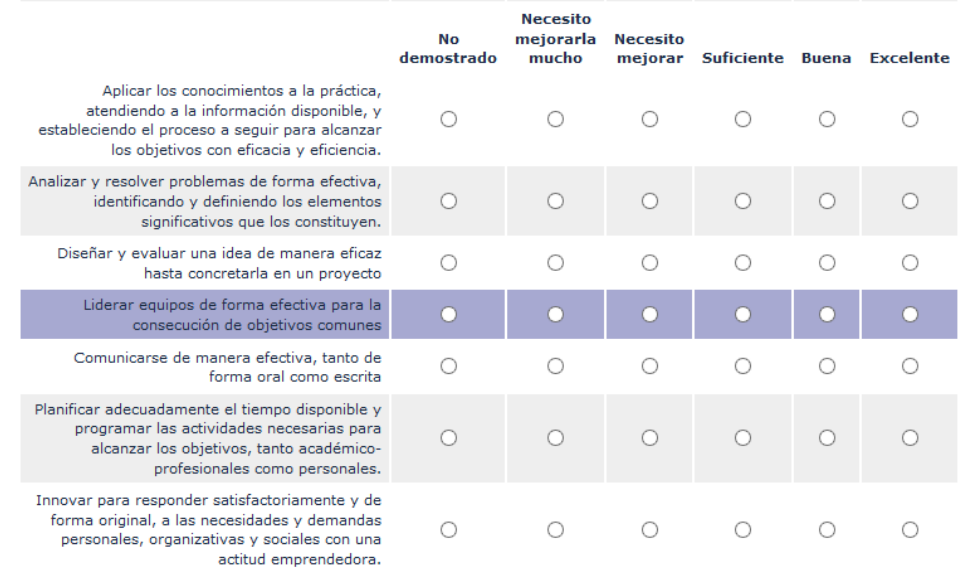

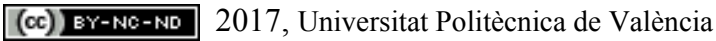


Figura 2. Ejemplo de parte del cuestionario para profesionales

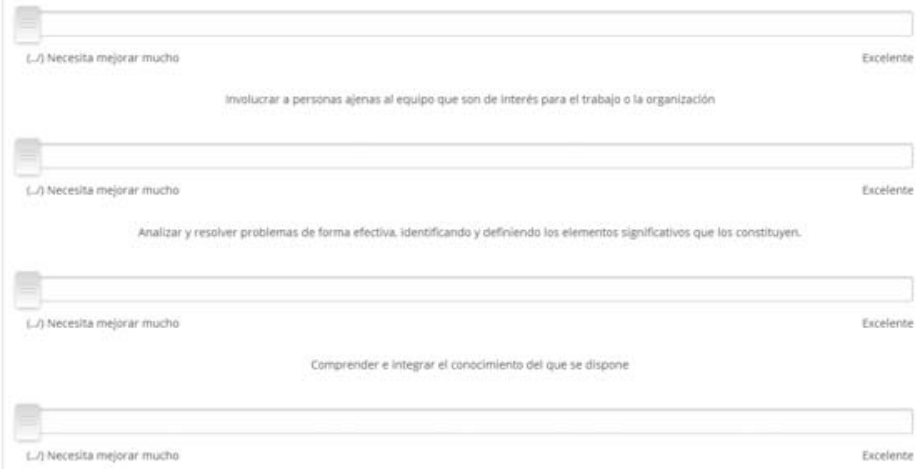

Para dar respuesta al objetivo 1 presentaremos la tabla de correlaciones de Pearson entre las competencias. Para el objetivo 2, realizaremos un análisis factorial exploratorio, con componentes principales, analizando correlaciones y con rotación ortogonal varimax (Hair et al., 1995; Losilla et al., 2005; Marin-Garcia, 2012; Marin-Garcia et al., 2013). El número de factores extraídos se basa en el criterio de eigenvalue $>1$, el análisis de pendiente de número de componentes y el criterio de varianza extraída superior al $80 \%$.

\section{Resultados}

En la Tabla 2 podemos apreciar cómo, en la muestra de estudiantes universitarios, se utilizan todos los valores de las escalas. El valor promedio, una vez re-escalados los valores de 0 a 100, están en torno a 60-70 puntos. Es decir, la media está en la parte alta de la escala. En la muestra de profesionales (Tabla 3), existe un efecto suelo en todas las dimensiones (en algunas en la cuarta parte de la escala y en otras en la mitad). Los valores medios son bastante más altos en la muestra de profesionales que en la de estudiantes. Hecho, éste, que resulta lógico pues indica que los profesionales (elegidos entre los mejores en empresas con clara tendencia innovadora), tienen más desarrolladas estas competencias que los estudiantes de ingeniería.

\begin{tabular}{|l|l|r|r|r|r|r|r|}
\hline & N & Range & Minimum & Maximum & $\begin{array}{c}\text { Mean } \\
(0-100)\end{array}$ & $\begin{array}{c}\text { Mean } \\
(0-5)\end{array}$ & $\begin{array}{c}\text { Std. } \\
\text { Deviation }\end{array}$ \\
\hline A_compre & 128 & 5 & 0 & 5 & 67.60 & 3,38 & 1,095 \\
\hline A_aplic & 128 & 5 & 0 & 5 & 64.00 & 3,20 & 1,075 \\
\hline A_proble & 128 & 5 & 0 & 5 & 67.60 & 3,38 & 1,129 \\
\hline A_empren & 128 & 5 & 0 & 5 & 61.80 & 3,09 & 1,230 \\
\hline A_creat & 129 & 5 & 0 & 5 & 64.00 & 3,20 & 1,114 \\
\hline A_project & 128 & 5 & 0 & 5 & 60.40 & 3,02 & 1,167 \\
\hline A_leader & 128 & 5 & 0 & 5 & 70.20 & 3,51 & 1,143 \\
\hline A_teamw & 128 & 4 & 1 & 5 & 74.00 & 3,70 & 1,062 \\
\hline A_commu & 128 & 5 & 0 & 5 & 71.00 & 3,55 & 1,162 \\
\hline A_criti & 129 & 5 & 0 & 5 & 72.20 & 3,61 & 1,127 \\
\hline A_time & 128 & 5 & 0 & 5 & 64.80 & 3,24 & 1,195 \\
\hline
\end{tabular}

Tabla 2. Estadísticos descriptivos de la muestra de alumnos 


\begin{tabular}{|l|l|r|r|r|r|r|}
\hline & N & Range & Minimum & Maximum & Mean & Std. Deviation \\
\hline A_compre & 45 & 75 & 25 & 100 & 77,44 & 14,692 \\
\hline A_aplic & 45 & 45 & 55 & 100 & 78,53 & 11,177 \\
\hline A_proble & 45 & 48 & 52 & 100 & 79,04 & 12,411 \\
\hline A_empren & 45 & 75 & 25 & 100 & 71,98 & 16,479 \\
\hline A_creat & 45 & 75 & 25 & 100 & 75,02 & 15,775 \\
\hline A_project & 45 & 75 & 25 & 100 & 74,76 & 14,808 \\
\hline A_leader & 45 & 75 & 25 & 100 & 71,84 & 10,247 \\
\hline A_teamw & 45 & 45 & 55 & 100 & 82,11 & 14,038 \\
\hline A_commu & 45 & 71 & 29 & 100 & 76,38 & 12,374 \\
\hline A_criti & 45 & 50 & 50 & 100 & 75,47 & 14,177 \\
\hline A_time & 45 & 55 & 45 & 100 & 72,04 & \\
\hline
\end{tabular}

Tabla 3. Estadísticos descriptivos de la muestra de profesionales

Las correlaciones son prácticamente todas significativas y de intensidad moderada alta. Destacando la existente entre emprendedurismo y creatividad.

\begin{tabular}{|c|c|c|c|c|c|c|c|c|c|c|c|}
\hline & $\begin{array}{c}\text { A_com } \\
\text { pre }\end{array}$ & $\underset{c}{\text { A_apli }}$ & $\underset{\text { le }}{\text { A_prob }}$ & $\underset{\text { ren }}{\text { A_emp }}$ & A_crea & $\begin{array}{l}\text { A_proj } \\
\text { ect }\end{array}$ & $\begin{array}{c}\text { A_lead } \\
\text { er }\end{array}$ & A_tea & $\underset{\mathrm{mu}}{\text { A_com }}$ & A_criti & A_time \\
\hline A_compre & 1 & ,488* & ,197 & ,719“ &, $500 "$ & ,499"* &, 556 & ,293 &, $516 “$ & $321^{\circ}$ & , $341^{*}$ \\
\hline A_aplic & ,663" & 1 & ,564" & ,475“ & ,440" &, 582 " & , 464" &, $538^{* *}$ & ,580" & ,444" & ,557" \\
\hline A_proble & ,539" & ,585* & 1 & $378^{\circ}$ & ,444" &, 524 &, $372^{*}$ & ,392" & ,226 & ,525 & ,522" \\
\hline A_empren & ,379" & ,457** & ,532* & 1 & ,786"* & ,651"* & $647^{* *}$ &, $357^{\star}$ &, $371^{*}$ & ,462" & $326^{*}$ \\
\hline A_creat & ,470" & , 471"* & ,632" & ,620" & 1 & ,704" & ,650" &, $374^{\star}$ & ,266 & ,531“ & ,442" \\
\hline A_project &, $486^{\prime \prime}$ & ,436" & ,609" & $608^{*}$ & ,609" & 1 &, $526^{* *}$ & $340^{*}$ & ,420" & $365^{\circ}$ & ,501“ \\
\hline A_leader & ,454" & ,524" &, $510^{* *}$ & ,490" &, 553 "* &, $510^{* *}$ & 1 & ,494" &, $503^{\prime \prime}$ &, 512 &, $513^{\prime \prime}$ \\
\hline A_teamw &, $514 "$ & ,462" & $365^{* \prime}$ & ,328" & ,455" & ,374" & ,589** & 1 & ,454" & , 188 & ,423“ \\
\hline A_commu & ,469" & ,438* & ,434" & ,418“ & , 475" & ,461** & ,628* & ,515" & 1 & ,244 & $371^{*}$ \\
\hline A_criti & ,431" & ,447** & ,625* & ,522" & ,617** &, 574 & ,481"* & , $447^{* *}$ & ,433" & 1 & ,576" \\
\hline A_time & ,386" & ,452"* & ,399" & ,350" & $405^{*}$ &, $216^{*}$ & , 405" & 276 & ,390" & $413^{* *}$ & 1 \\
\hline
\end{tabular}

Tabla 4. Matriz de correlaciones. Diagonal inferior correlaciones para muestra de estudiantes y diagonal superior muestra de profesionales. ** correlación significativa 0.01 (2-tailed), * correlación significativa 0.05 (2-tailed).

Los estadísticos de adecuación de las muestras al análisis factorial exploratorio tienen unos valores excelentes (Kaiser-Meyer-Olkin Measure of Sampling Adequacy: 0.91 para estudiantes y 0.83 para profesionales. En ambos casos el Bartlett's Test of Sphericity tiene una significación 0.000 ).

Con el criterio de eigen value mayor que 1, tanto para estudiantes como para profesionales, se extraería un solo factor (no se considerarían variables independientes sino indicadores de un mismo constructo latente), el cual explicaría un $53 \%$ de la varianza de la muestra de estudiantes y un 51.6 de la de profesionales.

Analizando los gráficos de valores propios (Figura 3), en la muestra de estudiantes existe un cambio de pendiente notable con dos factores y luego uno más sutil al pasar a 5 . En la muestra de profesionales, el segundo cambio se produce con cuatro factores.

\begin{tabular}{|l|l|}
\hline Estudiantes & Profesionales \\
\hline
\end{tabular}




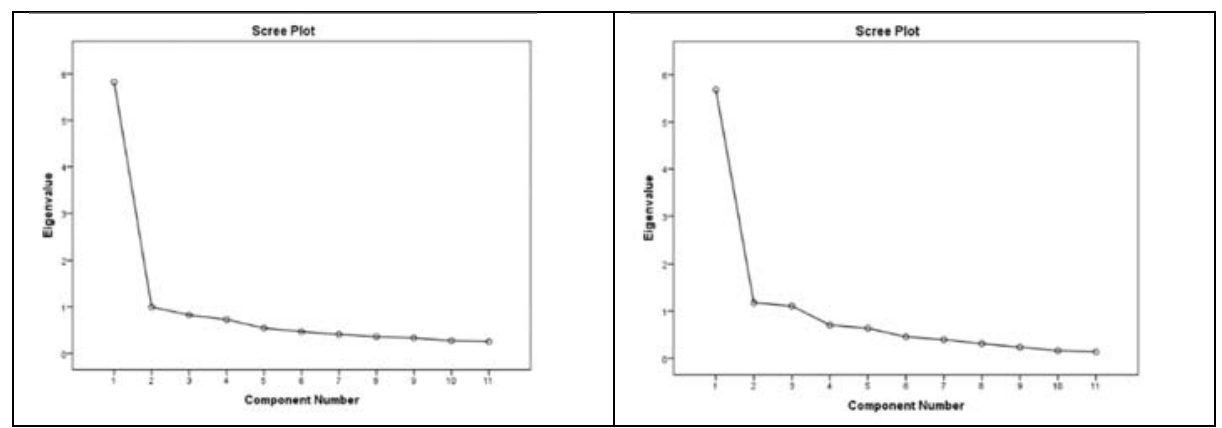

Figura 3.Grafico de valores propios en función de numero de factores extraídos.

Por último, con 5 factores se explica un $81 \%$ de la varianza de la muestra de estudiantes y un $84.6 \%$ de la de profesionales.

Las soluciones factoriales rotadas se muestran en la Tabla 5. En ella podemos ver que existen diferencias en la forma en la que se agrupan las variables en lo factores. Estas diferencias pueden ser debidas a estructuras de correlación diferentes o a que la muestra de profesionales es excesivamente escasa y la propuesta mostrada pueda no ser estable cuando se amplíe o cambie la muestra.

\begin{tabular}{|c|c|c|c|c|c|c|c|c|c|c|}
\hline & \multicolumn{5}{|c|}{ Estudiantes } & \multicolumn{5}{|c|}{ Profesionales } \\
\hline & 1 & 2 & 3 & 4 & 5 & 1 & 2 & 3 & 4 & 5 \\
\hline A_compre & ,198 & ,269 & ,829 & , 173 & ,081 & ,651 & ,118 & ,619 &, 001 &,- 025 \\
\hline A_aplic & ,274 & ,232 & ,787 & 074 & ,261 & ,191 & ,237 &, 563 & ,597 & ,236 \\
\hline A_proble & ,581 & ,080 &, 463 & ,383 & , 199 & ,162 & ,382 &,- 016 & ,784 & , 190 \\
\hline A_empren & ,814 & ,227 & ,141 & ,043 & ,195 & ,881 & ,155 & ,219 & , 171 & 100 \\
\hline A_creat & ,626 & ,280 & ,184 & ,428 & ,206 & ,826 & ,281 &,- 025 & ,279 & , 198 \\
\hline A_project & ,770 & ,242 & ,280 & ,227 &,- 122 & ,621 & ,071 & ,249 & ,611 & ,056 \\
\hline A_leader & ,368 & ,727 & ,212 & ,147 & ,192 & ,595 &, 460 & ,280 &,- 029 & ,432 \\
\hline A_teamw &,- 012 & ,709 & ,337 &, 501 &,- 053 & , 162 & , 070 & ,236 & ,236 & ,893 \\
\hline A_commu & ,322 & ,775 & ,184 &,- 002 & ,211 & ,141 & , 125 & ,855 & , 108 & ,258 \\
\hline A_criti & ,465 & ,171 &, 160 & ,725 & ,236 & ,295 & ,866 & ,068 & ,203 &,- 040 \\
\hline A_time & ,120 & ,193 & ,222 & ,154 & ,903 & , 105 & ,678 & ,266 & ,380 & ,246 \\
\hline
\end{tabular}

Tabla 5. Cargas factoriales

\section{Conclusiones}

Los datos presentados no permiten dar una respuesta concluyente a nuestra pregunta. Por un lado, una sola dimensión que agrupara las 9 competencias transversales analizadas explicaría en torno al $50 \%$ de la varianza de los datos de ambas muestras. A pesar de ser la solución más parsimoniosa, quizás no sea una cantidad suficiente de varianza para el estudio del efecto de estas competencia sobre otras variables. Es probable que el modelo con 5 dimensiones (no 9 como aparecen en la propuesta oficial de la UPV) sea una buena solución de compromiso entre nivel de detalle y cantidad de variables a manejar. Sin embargo la estructura factorial no parece respetarse entre la muestra de profesionales y la de estudiantes universitarios.

Esta investigación presenta algunas limitaciones que la investigación futura debería intentar superar:

- El uso de escalas de medida diferentes para estudiantes y para profesionales. Esto puede ser uno de los motivos para que los resultados en ambas muestras sean diferentes (en mayo de 2017 hemos recogido datos de una muestra equivalente de alumnos con la escala de medida igual a las de los profesionales y hemos ampliado

(c)) EY-NG-ND 2017, Universitat Politècnica de València 
la muestra de profesionales. En una investigación futura compararemos los resultados).

- Tenemos pocos datos de profesionales (comentada solución en el punto anterior)

- El uso de mono-ítem para el diagnóstico de competencias, que presenta ciertas desventajas frente a modelos de medida multi-ítem (Fisher et al., 2016; GiraldoO'Meara et al., 2014; Nagy, 2002; Sarstedt et al., 2016)

- Los datos analizados proceden sólo de auto-diagnóstico y en el futuro habría que añadir datos de evaluación de profesores o de mandos o compañeros

- No se han contemplado las 12 competencias transversales UPV

\section{Agradecimientos}

GVA/20161004: Project of Conselleria d'Educació, Investigació, Cultura i Esport de la Generalitat Valenciana a través del proyecto "Validación de la competencia transversal de innovación mediante un modelo de Medida formativo"

FINCODA: "Project 554493-EPP-1-2014-1-FI-EPPKA2KA" (The European Cornission support for the production of this publication does not constitute an endorsement of the contents which reflects the views only of the authors, and the Commission cannot be held responsible for any use which may be made of the information contained therein).

\section{Referencias}

AVAP Agència Valenciana d'Avaluació i Prospectiva

ANECA Agencia Nacional de Evaluación de la Calidad y Acreditación

EUR-ACE European Network for the Acreditation of Engineering Education (ENAEE)

ABET Accreditation Board for Engineering and Technology, Inc. (EEUU)

Argos, J., \& Ezquerra, P. (2014). Universities and skills for employability. Procedia - Social and Behavioral Sciences, 290-296. doi:http://dx.doi.org/10.1016/j.sbspro.2014.08.002

Fisher, G., Russell, A. M., \& Gibbons, A. M. (2016). Developing and investigating the use of single-item measures in organizational research. Journal of Occupational Health Psychology, 21(1), 3-23. doi:http://dx.doi.org/10.1037/a0039139

Giraldo-O'Meara, M., Marin-Garcia, J. A., \& Martínez-Gómez, M. (2014). Validation of the jds satisfaction scales applied to educational university environments. Journal of Industrial Engineering and Management, 7(1), 72-99.

Hair, J. F., Anderson, R. E., Tatham, R. L., \& Black, W. C. (1995). Multivariate data analysis ( $4^{\mathrm{o}}$ ed.). New Jersey: Prentice Hall.

Losilla, J. M., Navarro, J. B., Palmer, A., Rodrigo, M. F., \& Ato, M. (2005). Análisis de datos. Del contraste de hipótesis al modelado estadístico. Barcelona: Edicions a Petició.

Marin-Garcia, J. A. (2012). ¿cómo medir el grado de exigencias psicológicas requeridas por el entorno educativo español en el alumno universitario? (how to measure the degree of psychological demands required for university students in spanish environment?). WPOM-Working Papers on Operations Management, 3(2), 58-70.

Marin-Garcia, J. A., Alfalla-Luque, R., \& Medina-López, C. (2013). Supply chain integration scales validation and benchmark values. Journal of Industrial Engineering and Management, 6(2), 423-440. doi:http://dx.doi.org/10.3926/jiem.517

McGourty, J., Shuman, L., Besterfield-Sacre, M., Atman, C., Miller, R., Olds, B., ... Wolfe, H. (2002). Preparing for abet ec 2000: Research-based assessment methods and processes. International Journal of Engineering Education, 18(2), 157-167. 
Miró, J., \& Jaume i Capó, A. (2010). Repositorio de actividades para enseñar competencias transversales. REDU. Revista de Docencia Universitaria, 8(1), 101-110. doi: $10.4995 /$ redu. 2010.6219

Nagy, M. S. (2002). Using a single-item approach to measure facet job satisfaction. Journal of Occupational \& Organizational Psychology, 75(1), 77-86. doi:Article

Passow, H. J. (2012). Which abet competencies do engineering graduates find most important in their work? Journal of Engineerig Education, 101(1), 95-118.

Patil, A., \& Codner, G. (2007). Accreditation of engineering education: Review, observations and proposal for global accreditation. European Journal of Engineering Education, 32(6), 639-651. doi:10.1080/03043790701520594

Sarstedt, M., Diamantopoulos, A., \& Salzberger, T. (2016). Should we use single items? Better not. Journal of Business Research, 69(8), 3199-3203. doi:http://dx.doi.org/10.1016/j.jbusres.2016.02.040

Shuman, L. J., Besterfield-Sacre, M., \& McGourty, J. (2005). The abet "professional skills" - can they be taught? Can they be assessed? Journal of Engineering Education, 94(1), 41-55.

Terry, R. E., Harb, J. N., Hecker, W. C., \& Wilding, W. V. (2002). Definition of student competencies and development of an educational plan to assess student mastery level. International Journal of Engineering Education, JAM-PDF(2), 225-235.

Tovar, E., \& Castro, M. (2007). Building common spaces in engineering education: A review from icece05. IEEE Transactions on Education, 50(1), 79-84. doi:10.1109/TE.2006.888908

Weiss, M., \& Aldridge, M. D. (2002). Assessing the effects of the abet/nsf/industry regional faculty workshop. International Journal of Engineering Education, 18(2), 245-253.

Williams, J. M. (2002). The engineering portfolio: Communication, reflection, and student learning outcomes assessment. International Journal of Engineering Education, $J A M-P D F(2), 199-207$. 